\title{
ANÁLISE DE RISCO SOBRE O IMPACTO DAS MUDANÇAS CLIMÁTICAS GLOBAIS NA DISTRIBUIÇÃO ESPACIAL DA PINTA PRETA DO MAMOEIRO NO BRASIL
}

\author{
MORAES, Willian Bucker ${ }^{1,4}$ \\ MORAES, Wanderson Bucker ${ }^{2,5}$ \\ COSMI, Fernando Carrara ${ }^{2}$ \\ JESUS JUNIOR, Waldir Cintra de ${ }^{2,6}$ \\ CECILIO, Roberto Avelino ${ }^{2}$ \\ VALADARES JÚNIOR, Ranolfo ${ }^{2}$ \\ SOUZA , Antônio Fernando de ${ }^{3}$
}

RESUMO: O mamoeiro apresenta ampla distribuição geográfica, o que demonstra a grande capacidade de adaptação a diferentes condições climáticas, sendo uma fruteira de clima tropical. As mudanças climáticas globais (MCG) previstas para as próximas décadas poderão alterar os problemas fitossanitários da cultura do mamoeiro, de modo que é necessário e urgente entender os impactos dessas MCG sobre as doenças de modo a evitá-los. O presente trabalho visou analisar possíveis impactos MCG sobre a pinta preta do mamoeiro no Brasil, através da elaboração de mapas de distribuição da doença feitos a partir dos cenários do IPCC. Os mapas mostram redução da área favorável à doença em relação ao clima atual para os cenários futuros (cenário A2 eB2). As reduções na incidência da doença serão mais pronunciadas para o cenário A2 que para o cenário B2. Esse fato provavelmente está relacionado à redução da umidade relativa média para níveis desfavoráveis à ocorrência da doença, ou seja, para valores abaixo de $70 \%$ e aumento da temperatura média acima de $27^{\circ} \mathrm{C}$.

Palavras chave: Aquecimento global. Doenças de planta. Sistema de informação geográfico.

\section{RISK ANALYSIS OF THE IMPACT OF GLOBAL CLIMATE CHANGE IN DISTRIBUTION OF BLACK SPOT OF PAPAYA IN BRAZIL}

SUMMARY: Papaya has a wide geographical distribution, which demonstrates the great adaptability to different climatic conditions, being fruit from a tropical climate. Global climate change (GCC) scheduled for the coming decades could alter the disease problems of papaya crop, so it is necessary and urgent to understand the impacts of these diseases on the GCC in order to avoid them. This study aimed to analyze possible impacts on the GCC black spot of papaya in Brazil, through the mapping of disease distribution made from the IPCC scenarios. The maps show a reduction in the favorable area for the disease in relation to both current climate for future scenarios (scenario A2 as for B2). The reductions in disease incidence will be more pronounced for the A2 scenario than for the B2 scenario. This fact is probably related to the reduction in relative humidity to levels

\footnotetext{
${ }^{1}$ Universidade Estadual Paulista Júlio de Mesquita Filho, Faculdade de Ciências Agronômicas de Botucatu, Departamento de Defesa Fitossanitária, Fazenda Experimental Lageado, Lageado, CEP 18610-307, Botucatu, SP, Brasil. E-mail: moraeswb@ hotmail.com

${ }^{2}$ Universidade Federal do Espírito Santo, Centro de Ciência Agrárias, Departamento de produção vegetal, Alto universitário s/n, 29500-000, Alegre, ES, Brasil. E-

mail: wbucker@yahoo.com.br; wcintra@cca.ufes.br; carrara1@hotmail.com; racecilio@yahoo.com.br ${ }^{3}$ Instituto Federal de Educação, Ciência e Tecnologia do Espírito Santo, Campus Santa Teresa, Rodovia ES 080, Km 21, São João de Petrópolis, CEP 29650-000, Santa Teresa, ES, Brasil. E-mail:antoniofs@ifes.edu.br ${ }^{4}$ Bolsista de Doutorada da CAPES

${ }^{5}$ Bolsista de Iniciação Científica do $\mathrm{CNPq}$

${ }^{6}$ Bolsista de Produtividade em Pesquisa do CNPq
} 
detrimental to the disease, ie to below $70 \%$ and average temperature rise above $27^{\circ} \mathrm{C}$.

Keywords: Global warming. Plant diseases. Geographic information system.

\section{INTRODUÇÃO}

O Brasil é um dos maiores produtores mundiais de mamão, responsável por aproximadamente $38 \%$ da produção mundial, e a região Nordeste é a maior produtora nacional, seguida pela região Sudeste. O mamoeiro é bastante vulnerável à ocorrência de epidemias de doenças, principalmente por apresentar: baixa variabilidade de materiais genéticos; continuidade temporal das plantas (por se tratar de cultura perene, as plantas estão expostas a infecções, ataques de insetos e distúrbios fisiológicos por longo período de tempo); continuidade espacial dos pomares (ocupa uma área muito grande, praticamente sem barreiras físicas e onde ocorre intenso tráfego de pessoas e veículos). Essa baixa variabilidade genética e a continuidade no tempo e no espaço conduzem a uma alta vulnerabilidade da cultura, com importantes consequiências para a sustentabilidade deste importante agronegócio.

A varíola ou pinta preta (Asperisporium caricae) do mamoeiro é uma das principais doenças da cultura e que compromete enormemente a produção e a qualidade do mamão no Brasil (LIBERATO ; ZAMBOLIM, 2002; VENTURA et al., 2003; ZAMBOLIM et al., 2005; SUZUKI et al., 2007). Ela ocorre com maior intensidade em condições de temperaturas entre 23 e $27^{\circ} \mathrm{C}$, com ventos fortes e altas precipitações pluviométricas. Essas condições favorecem o desenvolvimento das lesões e a dispersão dos esporos das folhas mais velhas, consideradas a principal fonte de inóculo, onde a doença incide inicialmente, disseminando-se posteriormente para as mais novas.

Estima-se que as mudanças climáticas globais (MCG) possam afetar o zoneamento agrícola, a produtividade das culturas e as técnicas de manejo, alterando o atual cenário da agricultura brasileira, em cada região, com consequiências econômicas, sociais e ambientais (EPA, 1989). Até então, poucos trabalhos foram publicados analisando o potencial impacto das mudanças climáticas globais nas doenças do mamoeiro. Assim, é urgente e necessário tal entendimento visando direcionar medidas mitigadoras de manejo. Pelo exposto, o trabalho teve como objetivo avaliar os possíveis impactos das mudanças climáticas globais sobre a pinta preta do mamoeiro no Brasil, com elaboração de mapas de distribuição da doença feitos a partir dos cenários do IPCC. 


\section{MATERIAL E MÉTODOS}

Para elaboração dos mapas atuais de distribuição espacial das áreas climáticamente favoráveis ao estabelecimento e desenvolvimento da pinta preta do mamoeiro, empregaram-se dados mensais médios de temperatura e umidade relativa do ar, obtidos do Climate Research Unit (CRU) (NEW et al., 2002). Os dados são referentes às médias históricas destas variáveis no período entre 1961 e 1990, disponíveis no formato matricial (grid) com células de 10’ x 10' de latitude e longitude.

Com relação às projeções futuras de temperatura média do ar e umidade relativa do ar, foram utilizadas as previsões dos desvios destas variáveis preditas por seis modelos disponibilizadas pelo IPCC, a saber: GFDL-R30, CCSR/NIES, CSIROMk2, CGCM2, ECHAM4 e HadCM3 (IPCC, 2007). Os dados de umidade relativa estão disponíveis somente pelo modelo HadCM3, sendo assim, foram utilizados dados originados de um único modelo para essa variável climática (JESUS JUNIOR et al., 2008)

Os desvios dos dados climáticos futuros de temperatura média do ar e umidade relativa do ar, foram centrados nas décadas de 2020, 2050 e 2080 (IPCC, 2007), de acordo com os cenários de emissões A2 e B2. O cenário A2 descreve um futuro mais heterogêneo onde a regionalização é dominante. O cenário B2 descreve um futuro no qual a ênfase está em soluções locais para a sustentabilidade econômica, social e ambiental. Assim, o cenário A2 pode ser considerado mais "pessimista", com maior emissão de gases de efeito estufa, e o B2, "otimista" em relação às mudanças.

O SIG (Sistema de Informações Geográficas) Idrisi 32 foi utilizado para a elaboração dos mapas. Devido às diferentes resoluções espaciais dos modelos disponibilizados pelo IPCC, os desvios dos dados climáticos futuros foram reamostrados utilizando o SIG Idrisi 32 para geração de mapas com resolução espacial de 10' x 10' de latitude e longitude. Visando a redução da variabilidade da simulação, foi realizada a média dos seis modelos para a obtenção dos mapas dos desvios da temperatura média mensal dos cenários futuros. Para tal, utilizou-se a ferramenta de análise espacial (operação aritmética) do Idrisi 32. Para obtenção das projeções futuras mensais de temperatura média do ar e umidade relativa, os mapas dos desvios futuros destas variáveis foram somados com os mapas atuais de temperatura e umidade relativa com o auxílio da ferramenta de operação aritmética do Idrisi 32.

Baseado na sobreposição dos mapas mensais de temperatura média e umidade relativa do período atual e futuro (2020, 2050 e 2080) de ambos cenários (A2 e B2), foram elaborados mapas da distribuição espacial da pinta preta do mamoeiro. Para a confecção dos mapas da distribuição espacial da doença utilizaram-se classes climaticamente favoráveis definidas com 
base em dados epidemiológicos do efeito da temperatura e umidade relativa do ar no desenvolvimento da pinta preta do mamoeiro. Para tanto, foi considerado que o desenvolvimento da doença é favorecido por temperaturas entre 23 e $27^{\circ} \mathrm{C}$ e umidade relativa acima de 70\% (LIBERATO ; ZAMBOLIM, 2002; VENTURA et al., 2003; VENTURA et al., 2004; ZAMBOLIM et al., 2005; SUZUKI et al., 2007). Conseqüentemente, regiões que apresentam temperaturas médias inferiores a $23^{\circ} \mathrm{C}$ ou superiores a $27^{\circ} \mathrm{C}$ ou umidade relativa média inferior a $70 \%$ foram consideradas desfavoráveis à doença.

\section{RESULTADO E DISCUSSÃO}

Com relação ao potencial efeito das MCG na cultura do mamoeiro, de um modo geral, mesmo com as mudanças climáticas previstas nos dois cenários (A2 e B2) e nos três períodos (2020, 2050 e 2080), supõe-se que a cultura não sofrerá grandes alterações, dado que a amplitude de crescimento do mamoeiro se enquadra dentro dos valores das mudanças. $\mathrm{O}$ que poderá ocorrer é que algumas áreas se tornarão mais aptas ao cultivo que outras, fato que poderá propiciar o surgimento e/ou maior desenvolvimento de algumas novas regiões de plantio.

Os impactos das mudanças climáticas sobre as doenças de plantas, podem se expressar em diferentes aspectos, podendo causar efeitos diretos e indiretos tanto sobre os patógenos quanto sobre as plantas hospedeiras e a interação de ambos (CHAKRABORTY, 2005). Entre os principais destacam-se os efeitos nos danos causados pelas doenças, na distribuição geográfica das doenças, na eficiência dos métodos de controle e nos demais organismos que interagem com a planta (CHAKRABORTY, 2005).

No tocante ao potencial impacto das MCG sobre a pinta preta do mamoeiro no Brasil, com base nos resultados obtidos verifica-se que no futuro haverá redução da área favorável à doença em relação ao clima atual, tanto para o cenário A2 (Figuras 1 e 2) quanto para o B2 (Figuras 3 e 4). Esse fato provavelmente está relacionado à redução da umidade relativa média para níveis desfavoráveis à ocorrência da doença, ou seja, para valores abaixo de 70\% e aumento da temperatura média acima de $27^{\circ} \mathrm{C}$. As reduções na incidência da doença serão mais pronunciadas para o cenário A2 que para o cenário B2 (Figuras 1 a 4).

Os efeitos das mudanças climáticas sobre os danos causados pelas doenças são determinados pelas interações de um grande número de fatores que, direta ou indiretamente, influenciam a ocorrência e a intensidade das doenças. Entre os fatores diretos, as alterações da fisiologia e da morfologia da planta hospedeira, pelo aumento no teor de CO2, podem influir na interceptação da luz e precipitação, modificar a estrutura da parte aérea da planta e, 
conseqüentemente, o microclima, influenciando os componentes epidemiológicos das doenças (GHINI, 2005a).

Adicionalmente, todas as modalidades de controle de doenças de plantas são afetadas pelas condições climáticas (ATKINSON, 1993). Alterações na precipitação, por exemplo, quanto à duração, intensidade e frequiência de chuvas, têm efeito no controle químico - se ocorrerem no período pós-aplicação, muitos fungicidas, por exemplo, podem ter sua eficácia comprometida. Para o controle biológico das doenças, as alterações na composição da atmosfera podem modificar as comunidades da microbiota, da filosfera e rizosfera (MANNING; TIEDMANN, 1995).

O cenário A2 prevê maiores reduções na umidade relativa em comparação ao cenário B2, o que pode resultar em condições desfavoráveis ao desenvolvimento da pinta preta. Além disso, observa-se que a redução da área favorável à doença será gradativa para as três décadas estudadas, nos dois cenários considerados, ou seja, a proporção de área favorável à doença é reduzida gradativamente com o transcorrer das décadas de 2020 a 2080. Vale ressaltar, entretanto, que apesar disso, extensas áreas ainda continuarão favoráveis à ocorrência da doença, particularmente no Estado do Espírito Santo, um dos principais produtores de mamão do Brasil. Este fato implicará na necessidade de adoção de medidas adequadas de controle, particularmente nos meses mais favoráveis ao desenvolvimento da doença.

Nesse sentido, Jesus Junior et al. (2003, 2004, 2007b) discutiram vários critérios epidemiológicos que podem ser utilizados na tomada de decisão quanto ao manejo de doenças de plantas. De acordo com os autores, é possível racionalizar todas as estratégias de manejo desde que as mesmas sejam implementadas em momento adequado, levando em consideração cada componente do triângulo de doença (patógeno, hospedeiro e ambiente). 


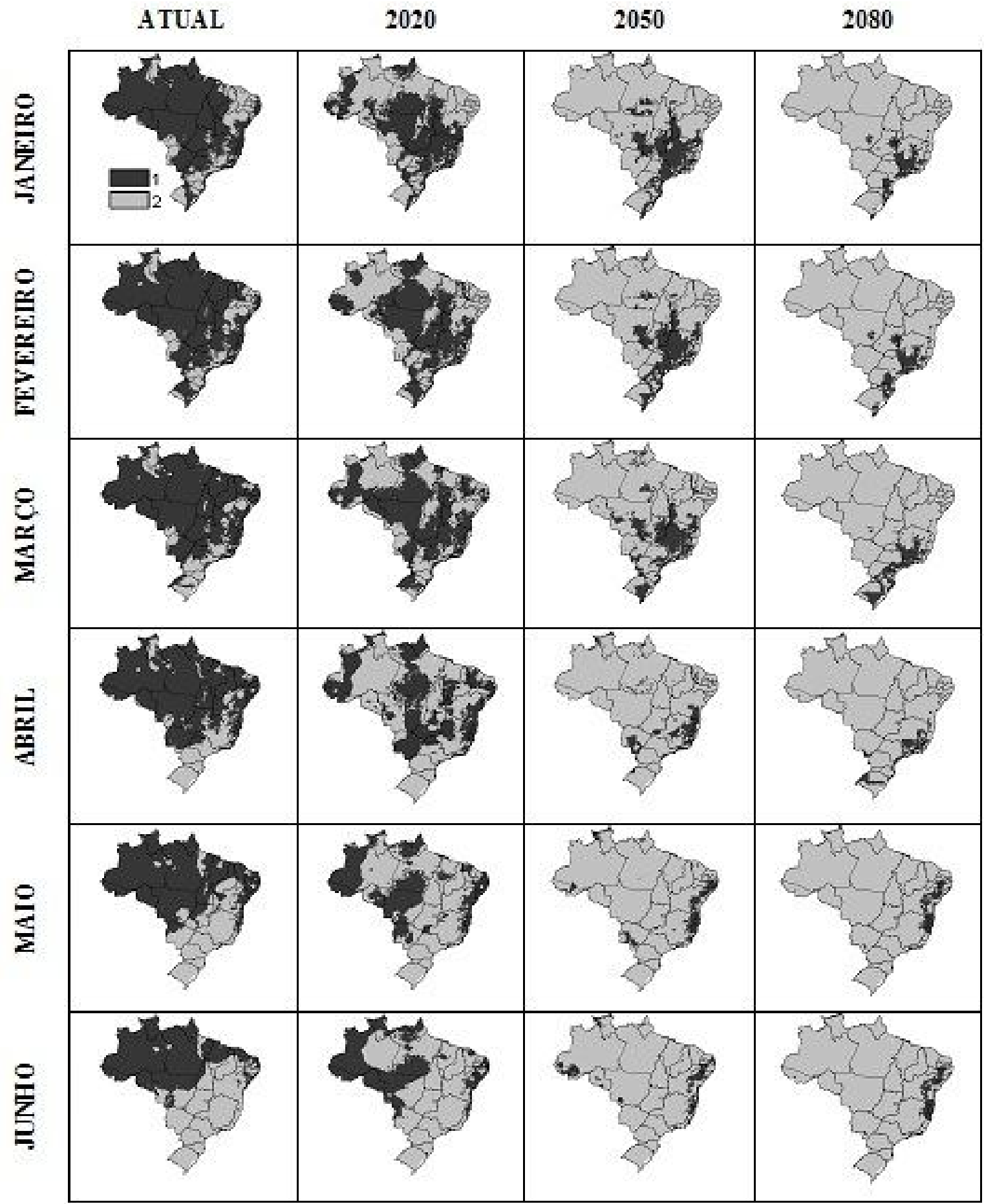

Favorável

Desfavorável

Figura 1. Mapa de risco climático de ocorrência da pinta preta do mamoeiro (Asperisporium caricae) no Brasil, para os meses de janeiro a junho, no período atual (média de 1961 a 1990) e futuro $(2020,2050$ e 2080) para o cenário A2 


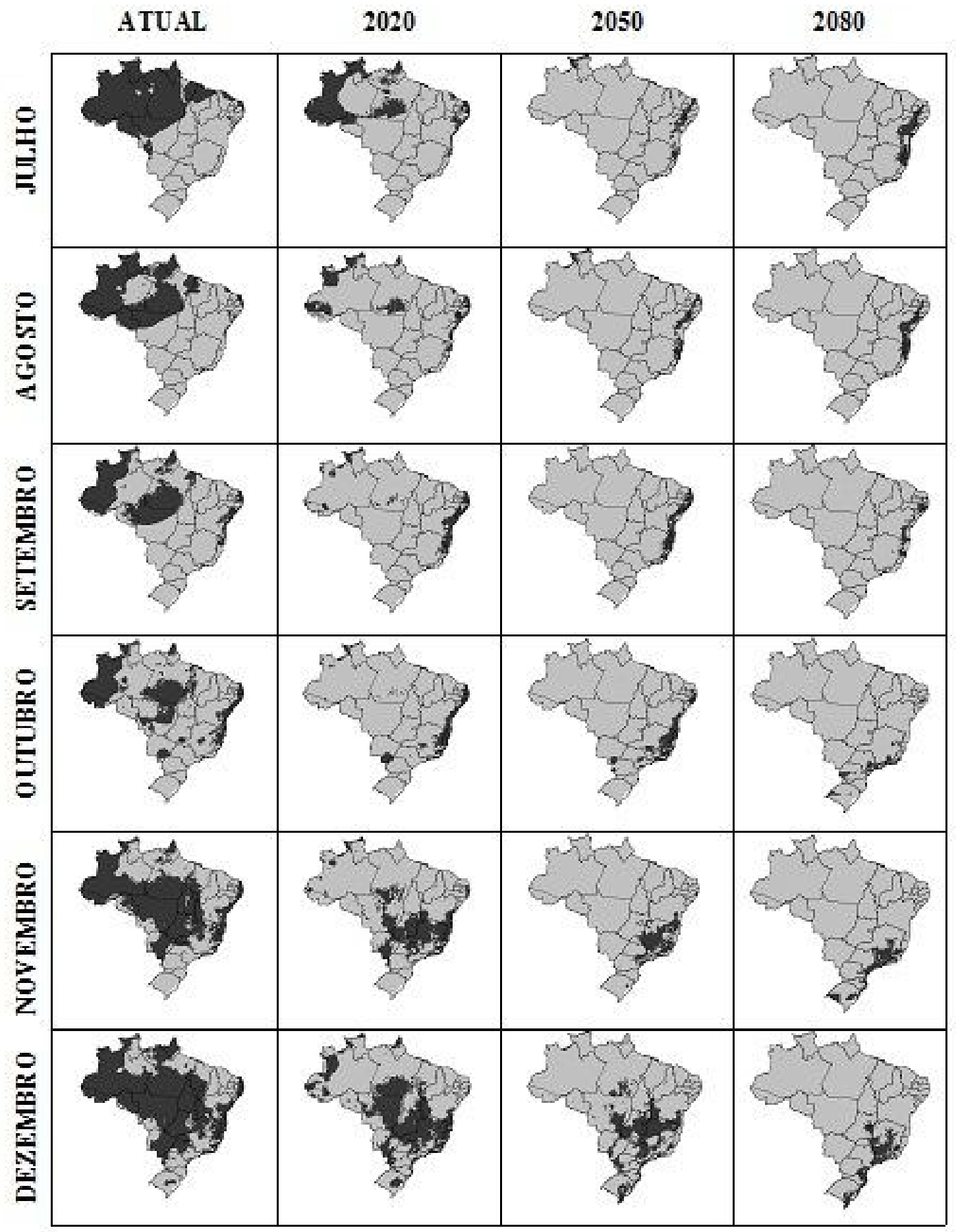

$\square$ Favorável $\square$ Desfavorável

Figura 2. Mapa de risco climático de ocorrência da pinta preta do mamoeiro (Asperisporium caricae) no Brasil, para os meses de julho a dezembro, no período atual (média de 1961 a $1990)$ e futuro $(2020,2050$ e 2080) para o cenário A2. 


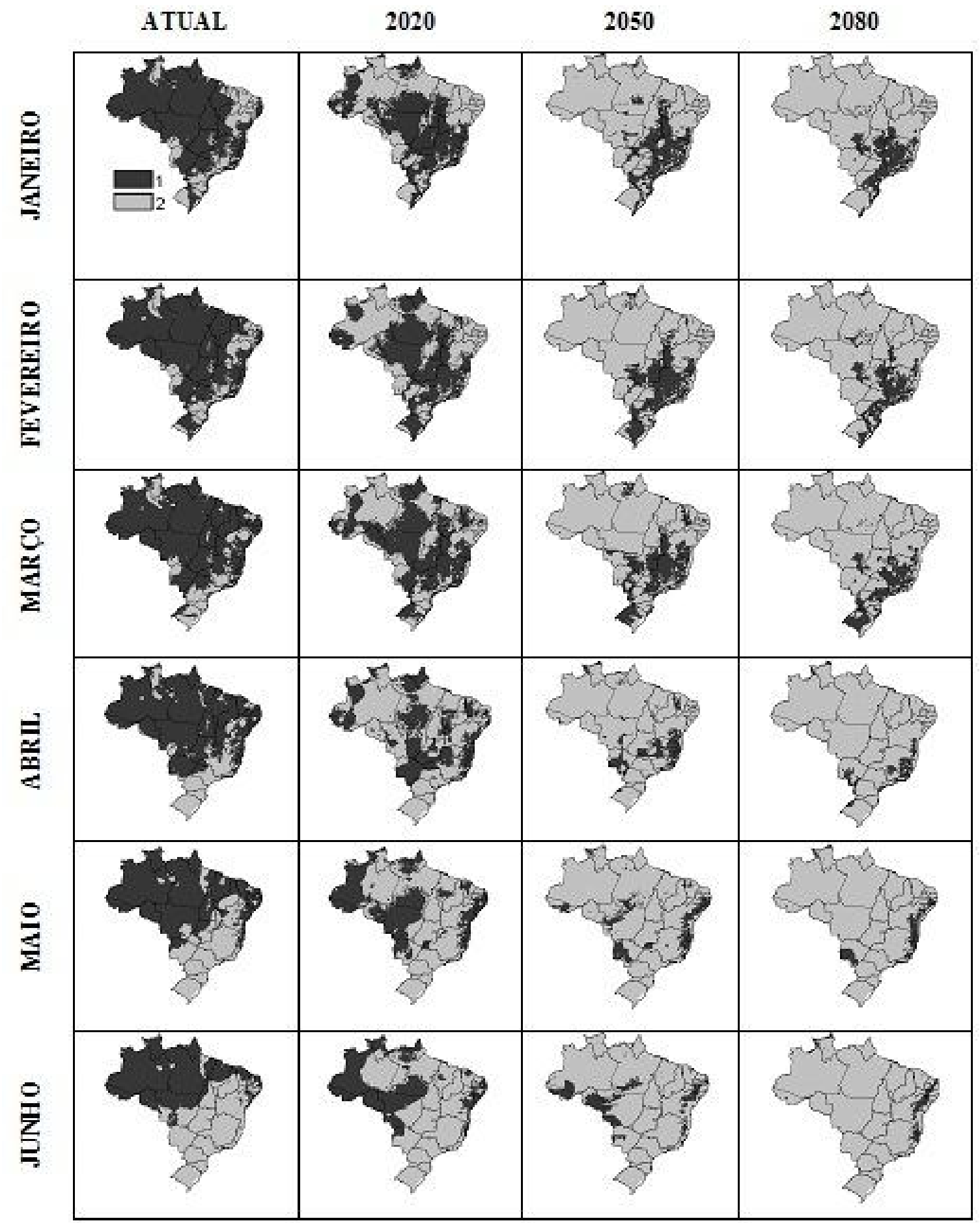

Favorável

Desfavorável

Figura 3. Mapa de risco climático de ocorrência da pinta preta do mamoeiro (Asperisporium caricae) no Brasil, para os meses de janeiro a junho, no período atual (média de 1961 a 1990) e futuro $(2020,2050$ e 2080) para o cenário B2. 


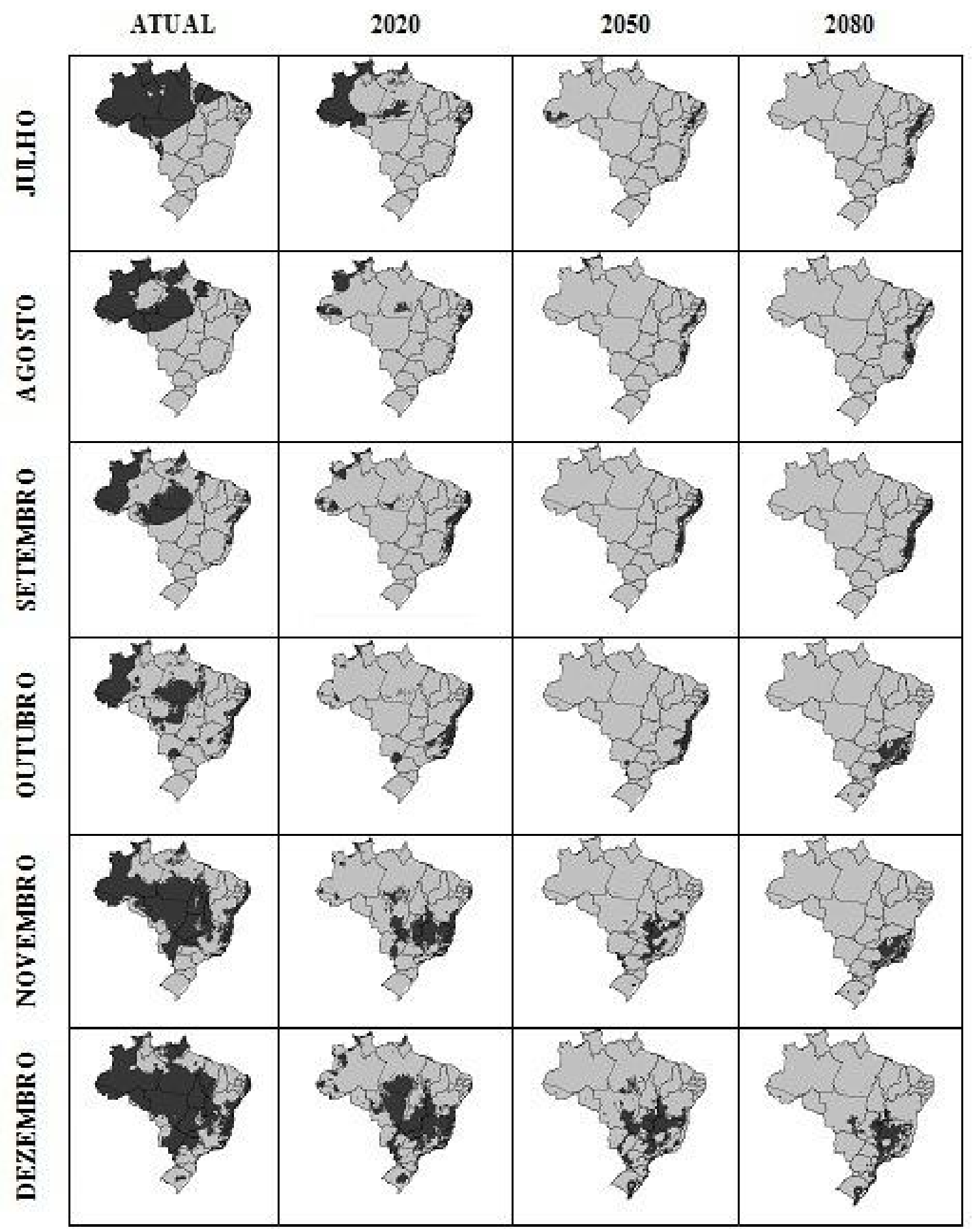

Figura 4. Mapa de risco climático de ocorrência da pinta preta do mamoeiro (Asperisporium caricae) no Brasil, para os meses de julho a dezembro, no período atual (média de 1961 a $1990)$ e futuro $(2020,2050$ e 2080) para o cenário B2. 


\section{CONCLUSÃO}

Dessa forma é possível verificar que as mudanças climáticas certamente afetarão o desenvolvimento do mamoeiro. Nas condições atuais, o mamoeiro é cultivado em várias regiões do país, mas nos cenários futuros, áreas de inaptidão poderão causar alteração da área cultivada comercialmente. Entretanto, tal situação poderá ser evitada e/ou amenizada com programas de melhoramento visando obtenção de materiais tolerantes às novas condições.

Consequentemente, o patógeno pode sofrer pressão de seleção em favor de linhagens mais adaptadas. As alterações nos parâmetros meteorológicos, especialmente a elevação da temperatura e a redução da umidade relativa podem ter efeitos adversos na ocorrência, severidade e padrão de distribuição das doenças do mamoeiro. Entretanto, há que se considerar que esta análise é complexa devido à possibilidade de efeitos compensatórios entre os fatores que favorecem ou desfavorecem as atividades dos patógenos, bem como de seus vetores, e afetam a predisposição das plantas a estes.

Assim, estudos multidisciplinares devem ser estimulados, visando englobar o maior número possível de variáveis de modo a reduzir as incertezas quanto aos potenciais impactos das mudanças climáticas globais, assim como direcionar possíveis medidas mitigadoras.

\section{REFERÊNCIAS}

ATKINSON, D. Global climate change: its implication for crop protection. British Crop Protection Council Monograph No. 56. BCPC, Surrey, UK. 1993.

CHAKRABORTY, S. Potential impact of climate change on plant-pathogen interactions. Australasian Plant Pathology, v.34, p.443-448, 2005.

ENVIRONMENTAL PROTECTION AGENCY. The potential effects of global climate change on the Unided States. Washington: EPA, 1989. Chapter 6. Agriculture (EPA-23005-89-050), p. 93-121.

GHINI, R. Mudanças climáticas globais e doenças de plantas. Jaguariúna: Embrapa Meio Ambiente, 2005a. 104p.

HAMADA, E.; GHINI, R.; GONÇALVES, R.R.V. Efeito da mudança climática sobre problemas fitossanitários de plantas: metodologia de elaboração de mapas. Engenharia Ambiental, v.3, p.73-85, 2006. 
HAMADA, E.et al. Efeito de mudanças climáticas globais sobre a distribuição espacial do número provável de gerações do bicho-mineiro do cafeeiro. CBAagro 2005. In:

CONGRESSO BRASILEIRO DE AGROMETEOROLOGIA, 14. Anais... 18 a 21 jul.2005, Unicamp, Campinas/SP.

HULME, M.; SHEARD, N. Cenários de alterações climáticas para o Brasil. Norwich: Climatic Research Unit, 1999. 6p.

IPCC. Climate change 2001: the scientific basis: summary for policymakers. Geneva: IPCC, 2001. 20p. Disponível em: 〈http://www.ipcc.ch/pub/spm22-01.pdf〉>. Acesso em: 10 jun. 2007.

IPCC. Climate change 2007: the physical science basis: summary for policymakers. Geneva: IPCC, 2007. 18p. Disponível em: $<$ http://www.ipcc.ch/SPM2feb07.pdf>. Acesso em: 10 jun. 2007.

JESUS JUNIOR, W.C.et al. Sistemas de auxílio à tomada de decisão no manejo de doenças de plantas. Revisão Anual de Patologia de Plantas, v.11, p.133-193, 2003.

JESUS JUNIOR, W.C.et al. Tomada de decisão no manejo de doenças de plantas. In: VALE, F.X.R.; JESUS JUNIOR, W.C.; ZAMBOLIM, L. (Eds.) Epidemiologia aplicada ao manejo de doenças de plantas. Belo Horizonte: Perffil, 2004. p.365-404.

JESUS JUNIOR, W.C.et al. Influência da temperatura e umidade relativa na distribuição espacial de Mycosphaerella fijiensis no Brasil. CONGRESSO BRASILEIRO DE AGROMETEOROLOGIA, 15. Anais... v.1, p.1-5, 2007a.

JESUS JUNIOR, W.C.et al. Critérios epidemiológicos para tomada de decisão no manejo de doenças de plantas. In: JESUS JUNIOR, W.C. et al. (Ed.). Atualidades em defesa

fitossanitária. Visconde do Rio Branco: Suprema, 2007b, v.1, p.307-326.

JESUS JUNIOR, W.C.et al. Worldwide geographical distribution of black sigatoka for banana: predictions based on climate change models. Scientia Agricola, v. 65, p. 40-53, 2008.

LIBERATO, J.R.; ZAMBOLIM, L. Controle das doenças causadas por fungos, bactérias e nematóides em mamoeiro. In: CONTROLE de doenças de plantas frutíferas. Visconde do Rio Branco: Suprema, 2002, v.2, p.1023-1169.

MANNING, W. J.; TIEDMANN, A. V. Climate change: potential effects of increased atmospheric carbon dioxide (CO2), ozone (O3), and Ultraviolet-B (UV-B) radiation on plant diseases. Environmental Pollution, v.88, p.219-245, 1995.

MARENGO, J.A. Mudanças climáticas globais e regionais: avaliação do clima atual do Brasil e projeções de cenários climáticos do futuro. Revista Brasileira de Meteorologia, v.16, p.1$18,2001$.

MORANDI, M.A.B.. Estimativas de áreas favoráveis ao desenvolvimento de pragas quarentenárias na citricultura do Estado de São Paulo. Fitopatologia Brasileira, v.30, p.175, 2005. 
SUZUKI, M.S.; ZAMBOLIM, L.; LIBERATO, J.R. Progresso de doenças fúngicas em correlação com variáveis climáticas em mamoeiro. Summa Phytopathologica, v.33, p.167$177,2007$.

VALADARES JÚNIOR, R.; JESUS JUNIOR, W.C.; CECÍLIO, R.A. Influência das mudanças climáticas na distribuição espacial da Mycosphaerella fijiensis no mundo. SIMPÓSIO BRASILEIRO DE SENSORIAMENTO REMOTO, 13. Anais ... v.1, p.443-447, 2007.

VALE, F.X.R.; JESUS JUNIOR, W.C.; ZAMBOLIM, L. Epidemiologia Aplicada ao Manejo de Doenças de Plantas. Belo Horizonte: Perfill, 2004, 532p.

VENTURA, J. A.; COSTA, H.; TATAGIBA, J. da S. Manejo das doenças do mamoeiro. In: MARTINS, D. dos S.; COSTA, A. de F.S. da (Ed.). A cultura do mamoeiro: tecnologias de produção. Vitória, ES: Incaper, 2003. p.231-308.

VENTURA, J. A.; COSTA, H.; TATAGIBA, J. da S. Papaya diseases and integrated control. In: NAQVI, S.A.M.H. (Ed.) Diseases of fruits and vegetables: diagnosis and management. Dordrecht: Kluwer Academic Publishers, 2004. p.201-268.

ZAMBOLIM, L.et al. Riscos e ameaças de patógenos com a introdução de material propagativo de mamão. In: MARTINS, D.S. Papaya Brasil: mercado e inovações tecnológicas para o mamão. Vitória, ES: Incaper, 2005. p.163-179. 